\title{
Biowaiver Study of Selected Solid Oral Prednisolone Products Available in Sri Lanka: Recommendations for Comparator Product Used in Biowaiver Testing
}

\author{
Hiruni Rathnayake ${ }^{1,2^{*}}$, Dhanusha Thambavita ${ }^{1}$, and Priyadarshani Galappatthy ${ }^{1}$ \\ ${ }^{1}$ Department of Pharmacology and Pharmacy, Faculty of Medicine, University of Colombo, Sri Lanka. \\ ${ }^{2}$ Department of Chemistry, Faculty of Science, University of Colombo, Sri Lanka.
}

e-mail: hirunirathnayake@gmail.com

\begin{abstract}
This post-marketing study on prednisolone products in Sri Lanka was conducted using BCS-based biowaiver procedures for performing in vitro bioequivalence studies. The market leader product with the highest availability was selected as the comparator in the absence of the innovator product. A validated UV/Visible spectrophotometric method was used to quantify the drug dissolution. Cumulative drug release profiles were determined in dissolution media at $\mathrm{pH}$ $1.2,4.5$, and 6.8 using the paddle apparatus. Although the two test products and the comparator met pharmacopoeial requirements for quality, they failed to meet in vitro biowaiver criteria. Acceptability of biowaiver study results depends on the performance of the comparator product. Information on the comparator product's bioequivalence to the innovator product was not available. This study revealed the difficulties in low and middle-income countries (LMICS) when conducting biowaiver studies for regulatory submission and highlights the necessity to have bioequivalence data and excipient information for the comparator product for better decision making. The study supports having an international standard comparator product used by LMICs when manufacturing and registering generic drug products.
\end{abstract}

KEYWORDS: Prednisolone, bioequivalence, biowaiver, BCS, pharmacopeia, dissolution

\section{INTRODUCTION}

A ssessment of therapeutic interchangeability of multisource (generic) pharmaceutical products with innovator products is essential during the registration process for generic drugs with regulatory authorities. Therapeutic equivalence is assessed through in vivo bioequivalence studies of generic and comparator products (1). In some cases, in vitro dissolution studies may assess therapeutic interchangeability as an alternative to in vivo studies. The Biopharmaceutics Classification System (BCS) of active pharmaceutical ingredients (APIs) has paved the way towards waiving the requirement of in vivo bioequivalence studies based on the solubility and permeability of the APIs (2). BCS-based dissolution studies are known as biowaiver studies $(1,2)$. Accordingly, BCS class 1 drugs with high solubility, high permeability, and BCS class 3 drugs with high solubility and low permeability are eligible for granting biowaiver (1).

The pharmaceutical industry in Sri Lanka is expanding in both government and private sectors, diversifying manufacturing and exporting pharmaceuticals. Most of the low- and middle-income countries (LMICs) like Sri Lanka are trying to penetrate the global market by improving the quality of medicine they produce. Currently, most LMICs only manufacture generic pharmaceuticals. Applying recent developments in BCS-based biowaiver studies to assess the quality and therapeutic interchangeability is relevant to evaluate the quality of eligible generic products. However, selecting a suitable comparator product for testing becomes challenging because it is difficult to find the innovator products in LMICs. The World Health Organization (WHO) guideline on comparator product selection recommends using the market leader as the comparator in such situations (1). Technically, this market leader product should be bioequivalent to the innovator; if not, then interpretation of in vitro biowaiver studies may be limited. This study highlights the issues related to selecting a suitable comparator product based on the results of a 
biowaiver study we conducted on prednisolone products. The current WHO recommendation for selection of a comparator product was used.

Prednisolone is classified as a BCS class 1 drug by the International Pharmaceutical Federation (3). Therefore, we conducted a post-marketing study using selected prednisolone 5-mg tablets in the Sri Lankan market to establish in vitro equivalence using BCS-based comparative dissolution studies.

\section{METHODS}

\section{Instrumentation and Equipment}

Dissolution studies were conducted using a dissolution tester with an auto-sampler (PTWS 610, Pharma Test, Germany), UV/VIS spectrophotometer(ORIONAQUAMATE 8000, Thermo Scientific), and a high-performance liquid chromatography (HPLC) system (Shimadzu, Japan) according to pharmacopoeial standards for quality testing of prednisolone tablet preparations.

\section{Reagents}

HPLC grade chemicals from Sigma Aldrich were used for HPLC analysis, and analytical grade chemicals from Sigma Aldrich were used for all the analytical procedures. Mili-Q water obtained from Smart2Pure Water Purification System was used for HPLC analysis, and de-aerated distilled water was used for pharmacopoeial buffer preparation.

\section{Materials}

Secondary reference standard of Prednisolone BP/ USP (Control No: SPMC/3/2012, Potency: 98.41\%, Date: 01/04/2016) donated by the State Pharmaceuticals Manufacturing Corporation (SPMC) was used for analysis of samples.

\section{Selection of Products}

The WHO guideline on comparator product selection was used to select a comparator product for the study $(1,4)$. The WHO provides several options for choosing a comparator product. Accordingly, the ideal comparator product is the innovator product with established quality, safety, efficacy, and national marketing authorization. If the innovator product is not available, the WHO recommends using the national market leader, choosing from the International List of Comparator Products, or choosing one approved in an $\mathrm{ICH}$-associated country $(1,4)$. Because the innovator and comparator products recommended by WHO guidance are not available in Sri Lanka, the market leader product was selected as the comparator product (4).

A small market study on the availability of prednisolone 5-mg products in Sri Lankan pharmacies was conducted according to the recommendations specified in the WHO/Health Action International (WHO/HAl) Project on Medicine Prices and Availability (5). Twenty pharmacies in and around the National Hospital Colombo, Sri Lanka, were randomly selected based on a convenience sampling method for this study. Among these pharmacies, the product with the most availability was identified as the market leader (manufactured by a local pharmaceutical company). Comparative tests were performed with the market leader and selected test products to assess the quality according to British Pharmacopoeia 2012 (BP) and the United States Pharmacopoeia (USP) 2011 (6, 7). The market leader met the pharmacopoeial quality standards and was thus designated the comparator product in the dissolution study. The comparator product was obtained from the National Hospital of Sri Lanka (NHSL), the country's main tertiary care hospital. The test products were selected during the market survey based on their price. The highest- and lowest-cost products (generic 1 and 4, respectively) were selected for testing to check for quality differences. All generic products were purchased from the local pharmacy.

\section{Pharmacopoeial Quality Testing}

The quality of products selected was determined using compendial identification, dissolution, and assay tests. Identification and assay tests were conducted according to $\mathrm{BP}$, and dissolution testing was conducted according to $\operatorname{USP}(6,7)$.

\section{Analytical Method Validation}

The analytical method was validated to determine the expected dissolution of prednisolone at specified time intervals in buffer media at $\mathrm{pH} 1.2,4.5$, and 6.8. A UV/VIS spectrophotometric method with direct measurement at $245 \mathrm{~nm}$ was developed and validated per the $\mathrm{ICH}$ guideline to quantify drug release during the dissolution test (8). Standard buffer media were prepared following the procedures given in the $\operatorname{USP}(7,8)$. The analytical range was determined based on the minimum percentage (10\% limit) of drug released within the first 5 minutes (lower limit, 10.0\%) and the maximum percentage $(110 \%$ limit) of drug released after complete dissolution. The analytical method was validated for linearity, accuracy, precision, specificity, lower limit of detection (LOD), and lower limit of quantification (LOQ). The degree of linearity was estimated by calculating the regression line using the least squares method, with a correlation coefficient $(r)$ of 0.995 (9). Accuracy was determined as the recovery percentage. Acceptance criteria were $\pm 20 \%$ of the expected value at the lower concentration and $\pm 15 \%$ at 
the higher concentration. Precision of the test system was determined by calculating the percentage relative standard deviation (\%RSD) of the measurements for each concentration; \%RSD less than or equal to $2.0 \%$ was used as the acceptance criteria for precision determination.

\section{In Vitro Comparative Dissolution Study}

The dissolution studies were conducted according to WHO recommendations for in vitro biowaiver studies for immediate-release solid oral dosage forms $(1,2)$. Dissolution tests were conducted for the comparator product and two generic products in three different buffer media prepared according to pharmacopoeial methods: pH 1.2 hydrochloric acid, pH 4.5 acetate, and pH 6.8 phosphate.

The buffer media (500-mL of each) were prepared in six amber-colored vessels in the dissolution apparatus, preheated to $40{ }^{\circ} \mathrm{C}$, and degassed using vacuum filtration to exclude dissolved air. The dissolution test was carried out for 12 dosage units from each product in a paddle apparatus and stirred at $75 \mathrm{rpm}$ and $37 \pm 0.5^{\circ} \mathrm{C}$. Samples $(4 \mathrm{~mL})$ were withdrawn from the auto-sampler at 5,10 , $15,20,30,45$, and 60-min time intervals. The same buffer at each interval replaced the volume. Absorbance was measured at $245 \mathrm{~nm}$ using the UV/VIS spectrophotometer, using the respective buffer solution as the blank. Dilution of drug concentration in the dissolution vessel due to sample withdrawal and refill at each sampling interval was corrected using the equation from Tsuji et al. (10).

The average percentage cumulative drug release at each time interval was calculated using a calibration curve, and comparative dissolution profiles were constructed. The dissolution profiles were compared using the similarity factor $\left(f_{2}\right)(1)$.

\section{RESULTS AND DISCUSSION Market Study Results}

Prednisolone products used in the study were registered in the National Medicines Regulatory Authority. There were 21 solid oral prednisolone 5-mg tablets and one product each of prednisolone $1 \mathrm{mg}$ and $25 \mathrm{mg}$. The availability of prednisolone tablets and the average price per tablet are illustrated in Figure 1. According to the criteria used for selecting a comparator product, the market leader with the highest availability (generic 7 in Fig. 1) was selected as the comparator product. The highest price was LKR 6.10 per tablet, and two products had the lowest price, LKR 1.44. The highest- and lowest-priced products (generic 1 and 4 , respectively) were selected as test products for the biowaiver study.

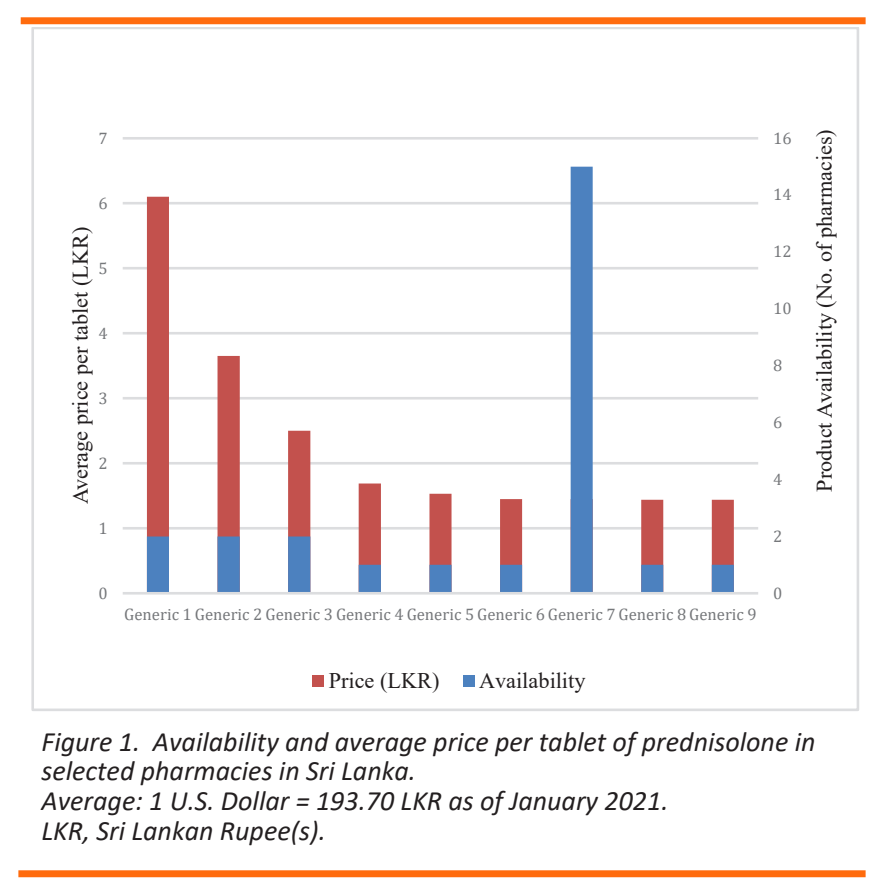

\section{Pharmacopoeial Quality Testing}

Results of pharmacopoeial quality testing for identification, dissolution, and assay tests are given in Table 1. According to these results, the products tested met the requirements of the pharmacopoeial standards.

\section{Analytical Method Validation}

The analytical method validation and acceptance criteria used for assessing each parameter are given in Table 2 . The analytical method validation results demonstrated linearity $\left(R^{2}=0.996-0.998\right)$ within the specified concentration range in all buffer media. Furthermore, results obtained for precision and accuracy met acceptance criteria.

\section{In Vitro Comparative Dissolution Study}

Cumulative drug release profiles for the tested prednisolone products in $\mathrm{pH} 1.2,4.5$, and 6.8 buffer media for 1 hour are given in Figures 2, 3, and 4, respectively.

In the $\mathrm{pH} 1.2$ buffer (Fig. 2), the comparator product released $>85 \%$ of the drug's labeled amount in 20 minutes, whereas generic 1 and 4 released $>85 \%$ within 15 minutes. Thus, the comparator product had rapid dissolution, and the test products had very rapid dissolution at $\mathrm{pH}$ 1.2. Thus, dissolution of the comparator and test products were different according to WHO recommendations. Accordingly, $f_{2}$ values of 19 and 46 were obtained for the comparator product versus generic 1 and 4 , respectively. An $f_{2}$ value of $50-100$ is required to be considered similar. Thus, the test products are not equivalent to the comparator product at $\mathrm{pH} 1.2$ medium. 
Table 1. Results of Pharmacopoeial Quality Testing of Selected Prednisolone 5-mg Products

\begin{tabular}{|c|c|c|c|c|}
\hline Test Type & Acceptance Criteria & Comparator & Generic 1 & Generic 4 \\
\hline Identification & Complied with the reference IR spectrum of prednisolone & Complied & Complied & Complied \\
\hline Dissolution & $\begin{array}{c}\text { Not less than } 70 \%(\mathrm{Q}) \text { of the labeled amount of prednisolone is } \\
\text { dissolved in } 30 \text { min. }\end{array}$ & $81.95 \%$ & $83.50 \%$ & $97.17 \%$ \\
\hline Assay & $90.0 \%<X<110.0 \%$ of the stated amount & $93.6 \%$ & $104.7 \%$ & $98.5 \%$ \\
\hline
\end{tabular}

$X$, labeled amount of prednisolone; $I R$, infrared.

Table 2. Results of Analytical Method Validation

\begin{tabular}{|c|c|c|c|c|}
\hline Parameter & Specification & pH 1.2 & pH 4.5 & pH 6.8 \\
\hline Range & - & 5-11 ppm & 5-11 ppm & 5-11 ppm \\
\hline Linearity & $\begin{array}{c}\text { Correlation coefficient }(r) \\
=0.995-1.0\end{array}$ & 0.996 & 0.998 & 0.998 \\
\hline LOD & - & $1.13 \mathrm{ppm}$ & $1.68 \mathrm{ppm}$ & $0.73 \mathrm{ppm}$ \\
\hline LOQ & - & $3.41 \mathrm{ppm}$ & $5.08 \mathrm{ppm}$ & $2.20 \mathrm{ppm}$ \\
\hline Accuracy & $\begin{array}{l}\text { Mean recovery is within } \pm \\
20 \% \text { at low concentrations } \\
\text { and } \pm 15 \% \text { at high } \\
\text { concentrations } \\
5 \mathrm{ppm} \\
8 \mathrm{ppm} \\
11 \mathrm{ppm}\end{array}$ & $\begin{array}{c}101.42 \% \\
101.36 \% \\
98.5 \%\end{array}$ & $\begin{array}{c}95.93 \% \\
102.25 \% \\
101.63 \%\end{array}$ & $\begin{array}{c}99.49 \% \\
101.18 \% \\
98.98 \%\end{array}$ \\
\hline Precision & $\begin{array}{c}\% \mathrm{RSD} \leq 2.0 \% \\
5 \mathrm{ppm} \\
8 \mathrm{ppm} \\
11 \mathrm{ppm}\end{array}$ & $\begin{array}{l}0.34 \% \\
0.95 \% \\
0.42 \%\end{array}$ & $\begin{array}{l}1.49 \% \\
1.93 \% \\
1.64 \%\end{array}$ & $\begin{array}{l}1.54 \% \\
1.55 \% \\
1.64 \%\end{array}$ \\
\hline Specificity & $\begin{array}{c}\text { Free of impurities and } \\
\text { excipients }\end{array}$ & No interfering peaks & No interfering peaks & No interfering peaks \\
\hline
\end{tabular}

$L O D$, lower limit of detection; $L O Q$, lower limit of quantification; RSD, relative standard deviation.

In the $\mathrm{pH} 4.5$ medium (Fig. 3), the comparator and generic 4 released $>85 \%$ of the drug's labeled amount in 20 minutes, showing rapid dissolution, whereas generic 1 released $>$ $85 \%$ in 5 minutes, showing very rapid dissolution. An $f_{2}$ value of 68 for the comparator versus generic 4 complied with the criteria for similarity; therefore, the similarity of dissolution profiles demonstrates in vitro equivalence of generic 4 and the comparator product at $\mathrm{pH} 4.5$. However, for generic 1 versus the comparator product, an $f_{2}$ value of 21 shows dissimilarity at $\mathrm{pH} 4.5$.

In $\mathrm{pH} 6.8$ (Fig. 4), both the comparator and generic 4 products released $>85 \%$ of the labeled amount of drug in 20 minutes (rapid dissolution), and generic 1 released $>85 \%$ in 5 minutes (very rapid dissolution). The $f_{2}$ values were 70 and 56 for generic 4 and 1 versus the comparator product, respectively. Thus, the two dissolution profiles were considered similar at $\mathrm{pH} 6.8$.

According to the dissolution profile comparison results, generic 4 had similar dissolution profiles with the comparator product in $\mathrm{pH} 4.5$ and 6.8 media. Generic 1 had a similar dissolution profile with the comparator product at $\mathrm{pH} 6.8$ only. The very rapid dissolution of generic 1 ( $>85 \%$ in 5 minutes) in all three $\mathrm{pH}$ levels may be due to an excipient effect; however, excipient information was not available.

Although the WHO recommends choosing the "leading" product on the market as a comparator product with proven quality attributes, availability or wide usage of such a product in a national market to be used as the key factor to select the comparator product poses several problems in LMICs. The comparator product used in our study was selected based on the market leader criteria in the absence of the innovator in the local market or a suitable national reference product. Proof of in vivo bioequivalence of the comparator product to the innovator or a reference listed product by a stringent regulatory authority was not available. Thus, using the market leader as the comparator may not be justified unless the market leader is bioequivalent to the innovator product. In the absence 
of such data, the innovator product may have to be used as the comparator product for biowaiver studies. The difficulties highlighted here will be faced by the generic drug applicants when registering their products in the future. Therefore, if the innovator product is not available, then a comparator product recommended by WHO in the International List of Comparator Products is preferable, because their quality, safety, and efficacy have been well documented, rather than using a market leader that may not have proven quality parameters (4).

In this study, the comparator was obtained from the National Hospital of Sri Lanka, where appropriate storage conditions are generally maintained. It may be preferable to purchase the comparator product directly from the drug manufacturer or local agent to use in a biowaiver study, along with the certificate of analysis of the batch requested from the manufacturer. Generic product manufacturers applying for biowaivers need to prove that differences in excipients will not affect the drug's dissolution profile. Therefore, the criteria used to select comparator products need further scrutiny by regulatory authorities to address practical issues faced by most developing countries when introducing a BCSbased biowaiver for generic product approval. It is also recommended to have a suitable mechanism to provide information required for generic drug applicants when planning biowaiver studies for regulatory submission.

The experience of this study highlights the practical difficulty of selecting a suitable market leader product as the comparator product in the absence of the innovator in the local market. The comparator product should meet additional criteria in addition to criteria laid down by the WHO guideline. Assistance with clear guidelines by the local regulatory agency is required to implement BCS-based biowaiver procedures in limited-resource countries. The difficulties faced during this study signal the need for an internationally accepted standard comparator product for use as a reference product in generic product approval processes and generic product manufacturing process. Löbenberg et al. reported similar obstacles in a study (with different drugs) in the Americas, and they concluded (11): "When coupled with the challenge of identifying a [comparator product] in different countries, the results of this study suggest the value of an international [comparator] as well as increased use of BCS approaches as means of either documenting bioequivalence or signaling the need for further in vivo studies." If such an international comparator is available, the national regulatory authorities could use these to develop secondary references on a regional or national basis for use as comparator products in their settings. An international comparator will help overcome practical difficulties the generic drug manufacturers face and support them to establish therapeutic equivalence of their product in an internationally accepted manner.

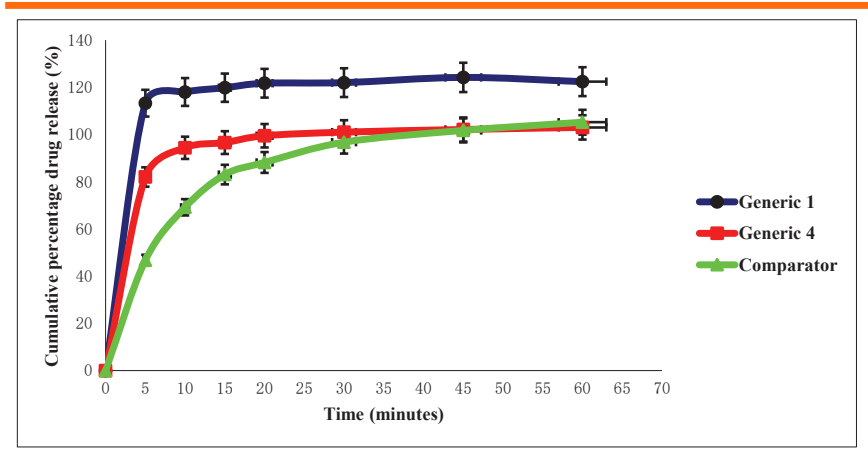

Figure 2. Dissolution profiles of prednisolone products at $p H$ 1.2.

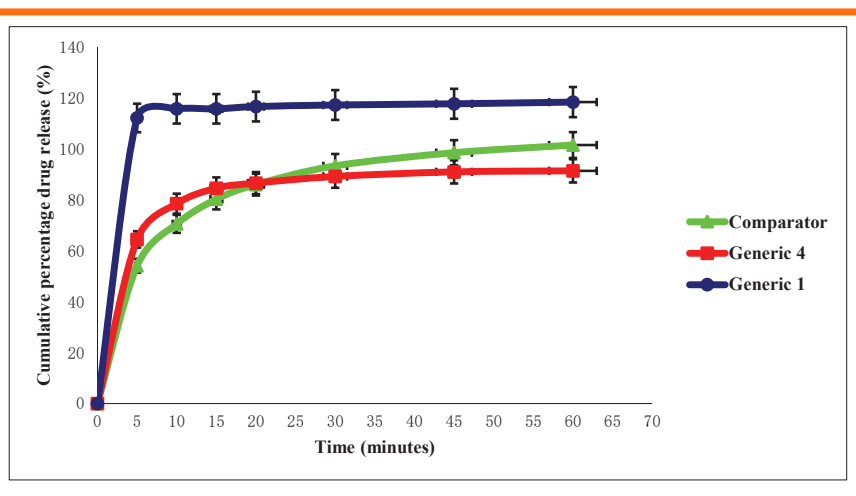

Figure 3. Dissolution profiles of prednisolone products at $\mathrm{pH} 4.5$.

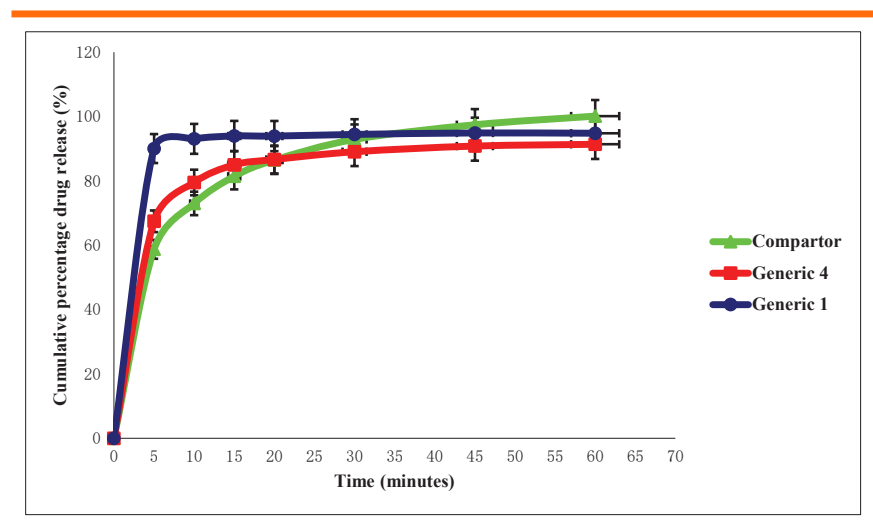

Figure 4. Dissolution profiles of prednisolone products at $\mathrm{pH} 6.8$. 


\section{CONCLUSIONS}

In this study of prednisolone 5-mg tablets, the comparator product and two test products were pharmaceutically equivalent according to relevant pharmacopoeial quality specifications. The two test products used in this study failed to meet in vitro dissolution criteria for BCS-based biowaiver. The comparator product did not have evidence to demonstrate bioequivalence with the innovator product. We recommend that the local drug regulatory authority provide a list of approved comparator/ reference products with proven bioequivalence data and necessary excipient information to be used for biowaiver studies submitted for regulatory approvals. Adopting an international standard comparator product for bioequivalence studies should be a key focus area for the WHO and other relevant authorities to address the issues related to identifying a suitable comparator product for use in studies to demonstrate therapeutic equivalence of the generic products.

\section{ACKNOWLEDGEMENTS}

We thank the State Pharmaceutical Manufacturing Corporation (SPMC) Sri Lanka for providing the prednisolone reference standard and the National Hospital of Sri Lanka for donating the comparator product. We acknowledge Ms. D.M.D. Chitra and Ms. P. Nugegoda for their technical assistance.

\section{FUNDING}

The study was funded by the University of Colombo infrastructure development grant.

\section{CONFLICTS OF INTEREST}

The authors disclosed no conflicts of interest related to this article.

\section{REFERENCES}

1. WHO Expert Committee on Specifications for Pharmaceutical Preparations. WHO Technical Report Series, No. 992; World Health Organization: Geneva, 2015.

2. Waiver of in Vivo Bioavailability and Bioequivalance Studies for Immediate-Release Solid Oral Dosage Forms Based on a Biopharmaceutics Classification System; Guideance for Industry; U.S. Department of Health and Human Services, Food and Drug Administration, Center for Drug Evaluation and Research (CDER), U. S. Government Printing Office: Washington, DC, 2015.

3. Vogt, M.; Derendorf, H.; Krämer, J.; Junginger, H. E.; Midha, K. K.; Shah, V. P.; Stavchansky, S.; Dressman, J. B.; Barends, D. M. Biowaiver monographs for immediate release solid oral dosage forms: prednisone. J. Pharm. Sci. 2007, 96, 1480-1489. DOI: 10.1002/jps.20817.

4. WHO Expert Committee on Specifications for Pharmaceutical Preparations. Guidance on the Selection of Comparator Pharmaceutical Products for Equivalence Assessment of Interchangeable Multisource (Generic) Products; WHO Technical Report Series, No. 902, Annex 11; World Health Organization: Geneva, 2002.

5. World Health Organization and Health Action International, Measuring Medicine Prices, Availability, Affordability and Price Components, $2^{\text {nd }}$ ed. World Health Organization: Geneva, 2008.

6. Prednisolone Tablets. British Pharmacopoeia. The Stationary Office: London, 2012.

7. The United States Pharmacopoeia. The United States Pharmacopoeial Convention, Inc: Rockville, MD, 2011.

8. International Conference on Harmonisation of Technical Requirements for Registration of Pharmaceuticals for Human Use. Validation of Analytical Procedures: Test and Methodology (Q2)R1; ICH Harmonised Tripartite Guideline: Geneva, 2005.

9. Appendix 1 - ORA Validation and Verification Guidance for Human Drug Analytical Methods; No. ORA-LAB 5.4.5, Version 1.7; U.S. Department of Health and Human Services, Food and Drug Administration, Office of Regulatory Affairs, 2014, 17-19.

10. Tsuji, A.; Nakashima, E.; Hamano, S.; Yamana, T. Physicochemical properties of amphoteric beta-lactam antibiotics I: stability, solubility, and dissolution behavior of amino penicillins as a function of pH. J Pharm Sci. 1978, 67, 1059-1066. DOI: 10.1002/ jps.2600670810.

11. Löbenberg, R.; Chacra, N. B.; Stippler, E. S.; Shah, V. P.; DeStefano, A. J.; Hauck, W. W.; Williams, R. L. Toward global standards for comparator pharmaceutical products: case studies of amoxicillin, metronidazole, and zidovudine in the Americas. AAPS J. 2012, 14, 462-472. DOI: 10.1208/s12248-012-9350-9. 